\title{
Finite gauge transformations and geometry in extended field theory
}

\author{
N. Chaemjumrus and C. M. Hull \\ The Blackett Laboratory, Imperial College London, Prince Consort Road, \\ London SW7 2AZ, United Kingdom \\ (Received 22 March 2016; published 18 April 2016)
}

\begin{abstract}
The recently derived expressions for finite gauge transformations in double field theory with duality group $O(d, d)$ are generalized to give expressions for finite gauge transformations for extended field theories with duality group $S L(5, \mathbb{R}), S O(5,5)$ and $E_{6}$. The generalized metrics are discussed.
\end{abstract}

DOI: 10.1103/PhysRevD.93.086007

\section{INTRODUCTION}

String theory on a toroidal background gives rise to a double field theory, with fields depending on a doubled spacetime in which the periodic coordinates $x^{i}$ on the torus are supplemented by dual periodic coordinates $\tilde{x}_{i}$ conjugate to the winding numbers. Key features of double field theory (DFT) are that T-duality is manifest and the fields depend on all the doubled coordinates. The double field theory corresponding to the metric, $b$-field and dilaton of the bosonic string was derived from string theory in [1] to cubic order in the fields. The full theory has proved rather intractable, and much work has been done on a small subsector of the theory, obtained by imposing the "strong constraint," which locally implies that locally all fields and parameters depend on only half the doubled coordinates. The strongly constrained theory has been found to all orders in the fields [2,3], and is locally equivalent to the conventional field theory of metric, $b$-field and dilaton, and DFT reduces to the dualitycovariant formulation of field theory proposed by Siegel [4], and can be thought of as a formulation in terms of generalized geometry [5-11]. Further details on the history of DFT relevant here are given in [12] and reviews and further references are given in [13-15].

There has been much work on extending this to formulations of supergravity in which U-duality is manifest. Generalized geometry has a natural action of the continuous group $O(d, d)$ and in [16,17] this was generalized to extended geometries in which there is a natural action of a duality group $E_{d}$. Doubled geometry with extra coordinates conjugate to string winding modes then has a natural generalization to an extended geometry with extra coordinates conjugate to brane wrapping modes [18], with a natural action of $E_{d}$ duality transformations. Extended field theories (EFT) generalize strongly constrained DFT to a theory on an extended geometry that is covariant under $E_{d}$ U-duality transformations [18-37]. See [14] for a review and further references on EFT.

DFT was derived for toroidal backgrounds, but the resulting theory has a certain background independence [3], suggesting that DFT could be applicable to more general backgrounds. To address such questions requires a better understanding of transition functions and global structure, and for this one needs formulas for gauge transformations with finite parameters. The infinitesimal gauge transformations of DFT or EFT are given by the action of the so-called generalized Lie derivative. As this is given by the usual Lie derivative in the doubled or extended spacetime, it is tempting to think of these in terms of some generalization of a diffeomorphism in the doubled or extended spacetime. However, the gauge algebra is very different from that of diffeomorphisms in the extended space, and is in fact locally equivalent to the algebra of diffeomorphisms and $p$-form gauge transformations in the original (unextended) spacetime. There have been a number of papers seeking expressions for finite gauge transformations in DFT [15,38-43]. There are a number of problems with some of these proposals, as has been discussed in [41] and [12]. In particular, the proposal of [38] attempts to realize the gauge transformations as diffeomorphisms in the doubled space, with novel transformation properties for generalized tensors. This runs into difficulties as the gauge algebra is not that of diffeomorphisms [12], and realizing $b$-field transformations as diffeomorphisms of dual coordinates is problematic in general [41].

In [12], explicit forms were found for finite gauge transformations in DFT that have the correct gauge algebra, are in agreement with the finite transformations for the metric and $b$-field, and which make explicit contact with generalized geometry. The purpose of this paper is to extend these results to extended field theories with duality group $E_{d}$. We will consider the cases $E_{4}=S L(5, \mathbb{R})$, $E_{5}=S O(5,5)$ and $E_{6}$; we expect similar results will hold for $E_{7}$. While this paper was in preparation, the paper [44] appeared addressing the same question, but using an approach which appears to suffer from the same issues as the approach of [38].

\section{FINITE TRANSFORMATIONS FOR DOUBLE FIELD THEORY}

In this section, we review the results of [12]. Double field theory has fields on a doubled space-time $M$ with coordinates $X^{M}$, where the indices $M, N=1, \ldots 2 D$ transform 
covariantly under the action of $O(D, D)$. Indices are raised and lowered using the constant $O(D, D)$ invariant matrix $\eta_{M N}$ of the form

$$
\eta_{M N}=\left(\begin{array}{ll}
0 & 1 \\
1 & 0
\end{array}\right)
$$

The fields and gauge parameters satisfy the strong constraint (so that $\partial^{M} \partial_{M} A=0$ and $\partial^{M} A \partial_{M} B=0$ for any fields or parameters $A$ and $B$ ). It was shown in [2] that the strong constraint implies that, at least locally, all fields are restricted to a $D$-dimensional subspace that is null with respect to $\eta$.

Consider a patch $\mathcal{U}$ of $M$ with coordinates

$$
X^{M}=\left(\begin{array}{l}
x^{m} \\
\tilde{x}_{m}
\end{array}\right)
$$

where $m=1, \ldots, D$. A generalized vector $W^{M}$ transforms as a vector under $O(D, D)$ and decomposes as

$$
W^{M}=\left(\begin{array}{c}
w^{m} \\
\tilde{w}_{m}
\end{array}\right),
$$

under $G L(D, \mathbb{R})$. The strong constraint is solved by having all fields independent of $\tilde{x}_{m}$ so that

$$
\tilde{\partial}^{m}=0
$$

on all fields and parameters. Then the fields just depend on the coordinates $x^{m}$, parametrizing a $D$-dimensional patch $U$ (which can be thought of as the quotient of $\mathcal{U}$ by the isometries generated by $\partial / \partial \tilde{x}_{m}$ ).

The generalized Lie derivative

$$
\hat{\mathcal{L}}_{V} W^{M}=V^{P} \partial_{P} W^{M}+W^{P}\left(\partial^{M} V_{P}-\partial_{P} V^{M}\right)
$$

for $V^{M}(x), W^{M}(x)$ then gives

$$
\left(\hat{\mathcal{L}}_{V} W\right)^{m}=v^{p} \partial_{p} w^{m}-w^{p} \partial_{p} v^{m}=\mathcal{L}_{v} w^{m}
$$

and

$$
\begin{aligned}
\left(\hat{\mathcal{L}}_{V} W\right)_{m} & =v^{p} \partial_{p} \tilde{w}_{m}+\tilde{w}_{p} \partial_{m} v^{p}+w^{p}\left(\partial_{m} \tilde{v}_{p}-\partial_{p} \tilde{v}_{m}\right) \\
& =\mathcal{L}_{v} \tilde{w}_{m}+w^{p}\left(\partial_{m} \tilde{v}_{p}-\partial_{p} \tilde{v}_{m}\right)
\end{aligned}
$$

where $\mathcal{L}_{v}$ is the usual Lie derivative on $U$.

Under an infinitesimal transformation with parameter $V^{M}, W^{M}$ transforms as

$$
\delta W^{M}=\hat{\mathcal{L}}_{V} W^{M}
$$

giving

$$
\begin{gathered}
\delta w^{m}=\mathcal{L}_{v} w^{m} \\
\delta \tilde{w}_{m}=\mathcal{L}_{v} \tilde{w}_{m}+w^{p}\left(\partial_{m} \tilde{v}_{p}-\partial_{p} \tilde{v}_{m}\right) .
\end{gathered}
$$

It will be convenient to rewrite the components of the generalized vector $W$ as

$$
w=w^{m} e_{m} \quad \tilde{w}_{(1)}=\tilde{w}_{m} e^{m},
$$

where $e_{m}=\partial / \partial x^{m}$ and $e^{m}=d x^{m}$ are the coordinate bases for $T U$ and $T^{*} U$, respectively. Then the generalized vector can be written as

$$
W=w \oplus \tilde{w}_{(1)} .
$$

Under an infinitesimal transformation with parameter $V$, these transform as

$$
\begin{gathered}
\delta_{V} w=\mathcal{L}_{v} w \\
\delta_{V} \tilde{w}_{(1)}=\mathcal{L}_{v} \tilde{w}_{(1)}-\imath_{w} d \tilde{v}_{(1)},
\end{gathered}
$$

where $\mathcal{L}_{v}$ is a Lie derivative on patch $U$.

Next, we introduce a gerbe connection $B_{(2)}$ on $U$,

$$
B_{(2)}=\frac{1}{2} B_{m n} e^{m} \wedge e^{n},
$$

which transforms under the gauge transformation as

$$
\delta_{V} B_{(2)}=\mathcal{L}_{v} B_{(2)}+d \tilde{v}_{(1)} .
$$

Then

$$
\hat{w}_{(1)}=\tilde{w}_{(1)}+l_{w} B_{(2)},
$$

transforms as

$$
\delta_{V} \hat{w}_{(1)}=\mathcal{L}_{v} \hat{w}_{(1)}
$$

and so is a 1-form on $U$, and is invariant under the $\tilde{v}$ transformations. Then

$$
\hat{W}=w \oplus \hat{w}_{(1)}
$$

is a section of $\left(T \oplus T^{*}\right) U$.

The finite transformation of $\hat{W}$ is given by

$$
w^{\prime}\left(x^{\prime}\right)=w(x) \quad \hat{w}_{(1)}^{\prime}\left(x^{\prime}\right)=\hat{w}_{(1)}(x),
$$

where $x^{\prime}(x)=e^{-v^{m} \partial_{m}} x$. Using the finite transformation of the coordinate bases 


$$
e_{m}^{\prime}\left(x^{\prime}\right)=e_{n}(x) \frac{\partial x^{n}}{\partial x^{\prime m}} \quad e^{\prime m}\left(x^{\prime}\right)=e^{n}(x) \frac{\partial x^{\prime m}}{\partial x^{n}},
$$

the finite transformation of the components of $\hat{W}$ are then

$$
w^{\prime m}\left(x^{\prime}\right)=w^{n}(x) \frac{\partial x^{\prime m}}{\partial x^{n}} \quad \hat{w}_{m}^{\prime}\left(x^{\prime}\right)=\hat{w}_{n}(x) \frac{\partial x^{n}}{\partial x^{\prime m}} .
$$

The finite transformation of the gerbe connection can be taken to be

$$
B_{(2)}^{\prime}\left(x^{\prime}\right)=B_{(2)}(x)+d \tilde{v}_{(1)}(x),
$$

so that

$$
\hat{w}_{(1)}^{\prime}\left(x^{\prime}\right)=\tilde{w}_{(1)}^{\prime}\left(x^{\prime}\right)+t_{w^{\prime}} B_{(2)}^{\prime}\left(x^{\prime}\right) .
$$

This then gives the finite transformations of $\tilde{w}_{(1)}$ :

$$
\begin{aligned}
\tilde{w}_{(1)}^{\prime}\left(x^{\prime}\right) & =\hat{w}_{(1)}^{\prime}\left(x^{\prime}\right)-\imath_{w^{\prime}} B_{(2)}^{\prime}\left(x^{\prime}\right), \\
& =\hat{w}_{(1)}(x)-\imath_{w}\left(B_{(2)}(x)+d \tilde{v}_{(1)}(x)\right), \\
& =\tilde{w}_{(1)}(x)-\imath_{w} d \tilde{v}_{(1)}(x) .
\end{aligned}
$$

To summarize, the transformation of $W$ is given by

$$
\begin{gathered}
w^{\prime}\left(x^{\prime}\right)=w(x), \\
\tilde{w}_{(1)}^{\prime}\left(x^{\prime}\right)=\tilde{w}_{(1)}(x)-\imath_{w} d \tilde{v}_{(1)}(x),
\end{gathered}
$$

which implies the components $\left(w^{m}, \tilde{w}_{m}\right)$ transform as

$$
\begin{gathered}
w^{\prime m}\left(x^{\prime}\right)=w^{n}(x) \frac{\partial x^{\prime m}}{\partial x^{n}} \\
\tilde{w}_{m}^{\prime}\left(x^{\prime}\right)=\left[\tilde{w}_{p}(x)-w^{n}(x)\left(\partial_{n} \tilde{v}_{p}(x)-\partial_{p} \tilde{v}_{n}(x)\right)\right] \frac{\partial x^{p}}{\partial x^{\prime m}} .
\end{gathered}
$$

\section{FINITE TRANSFORMATIONS FOR EXTENDED FIELD THEORY}

\section{A. $S L(5, \mathbb{R})$ extended field theory}

In $S L(5, \mathbb{R})$ extended field theory [21,30], a generalized vector $W^{M}$ transforms as a $\mathbf{1 0}$ of $S L(5, \mathbb{R})$ where the indices $M, N=1, \ldots, 10$ label the $\mathbf{1 0}$ representation of $S L(5, \mathbb{R})$. It decomposes under $G L(4, \mathbb{R}) \subset S L(5, \mathbb{R})$ into a vector and 2 -form:

$$
W^{M}=\left(\begin{array}{c}
w^{m} \\
\tilde{w}_{m n}
\end{array}\right)
$$

where $m, n=1, \ldots, 4$ and $\tilde{w}_{m n}=-\tilde{w}_{n m}$. The coordinates in a patch $\mathcal{U}$ consist of 7 spacetime coordinates $y^{\mu}$, $\mu=0, \ldots, 6$, together with 10 internal coordinates $X^{M}$ transforming as a $\mathbf{1 0}$ of $S L(5, \mathbb{R})$. This decomposes under $G L(4, \mathbb{R}) \subset S L(5, \mathbb{R})$ as

$$
X^{M}=\left(\begin{array}{c}
x^{m} \\
\tilde{x}_{m n}
\end{array}\right)
$$

where, in a suitable duality frame, $x^{m}$ are the usual coordinates on $T^{4}$ and $\tilde{x}_{m n}$ are periodic coordinates conjugate to M2-brane wrapping numbers on $T^{4}$. Fields and gauge parameters depend on both $y^{\mu}$ and $X^{M}$, but we will suppress dependence on $y^{\mu}$ in what follows.

The strong constraint of $S L(5, \mathbb{R})$ EFT is given by

$$
\epsilon^{i M N} \epsilon_{i P Q} \partial_{M}(\ldots) \partial_{N}(\ldots)=0,
$$

where $(\ldots)$ represents fields and gauge parameters, and the indices $i, j=1, \ldots, 5$ label the fundamental representation 5 of $S L(5, \mathbb{R})$. An index $M$ can be regarded as an antisymmetric pair of indices $\left[m_{1} m_{2}\right]$, so that $\epsilon^{i M N}=$ $\epsilon^{i m_{1} m_{2} n_{1} n_{2}}$. The strong constraint can be solved such that the fields are independent of wrapping coordinates $\tilde{x}_{m n}$ so that

$$
\tilde{\partial}^{m n}(\ldots)=0
$$

where $\tilde{\partial}^{m n}=\frac{\partial}{\partial \tilde{x}_{u}}$. The gauge transformations of $\operatorname{SL}(5, \mathbb{R})$ extended field theory are given by the generalized Lie derivative, which is defined as

$\hat{\mathcal{L}}_{V} W^{M}=V^{N} \partial_{N} W^{M}-W^{N} \partial_{N} V^{M}+\epsilon^{i M N} \epsilon_{i P Q} \partial_{N} V^{P} W^{Q}$.

It is convenient to rewrite the components of the generalized vector as

$$
\begin{gathered}
w=w^{m} e_{m}, \\
\tilde{w}_{(2)}=\frac{1}{2 !} \tilde{w}_{m n} e^{m} \wedge e^{n} .
\end{gathered}
$$

Then the generalized vector $W$ is

$$
W=w \oplus \tilde{w}_{(2)} .
$$

Under a gauge transformation with gauge parameter $V$, a generalized vector $W$ transforms as

$$
\delta_{V} W=\hat{\mathcal{L}}_{V} W
$$

This decomposes into

$$
\begin{gathered}
\delta_{V} w=\mathcal{L}_{v} w \\
\delta_{V} \tilde{w}_{(2)}=\mathcal{L}_{v} \tilde{w}_{(2)}-\imath_{w} d \tilde{v}_{(2)},
\end{gathered}
$$


where $\mathcal{L}_{v}$ is the ordinary Lie derivative with a parameter $v$.

Next we introduce a gerbe connection $C_{(3)}$,

$$
C_{(3)}=\frac{1}{3 !} C_{m n p} e^{m} \wedge e^{n} \wedge e^{p},
$$

which transforms under a gauge transformation as

$$
\delta_{V} C_{(3)}=\mathcal{L}_{v} C_{(3)}+d \tilde{v}_{(2)}
$$

This allows us to define $\hat{w}_{(2)}$ by

$$
\hat{w}_{(2)}=\tilde{w}_{(2)}+\iota_{w} C_{(3)} .
$$

Under a gauge transformation, this transforms as a 2-form

$$
\delta_{V} \hat{w}_{(2)}=\mathcal{L}_{v} \hat{w}_{(2)}
$$

and is invariant under the $\tilde{v}$ transformations. Therefore, $\hat{W}=w \oplus \hat{w}_{(2)}$ is a section of $\left(T \oplus \Lambda^{2} T^{*}\right) U$. This allows us to immediately write down the finite transformation of $\hat{W}$, which is given by

$$
\begin{gathered}
w^{\prime}\left(x^{\prime}\right)=w(x), \\
\hat{w}_{(2)}^{\prime}\left(x^{\prime}\right)=\hat{w}_{(2)}(x),
\end{gathered}
$$

where $x^{\prime}=e^{-v^{m} \partial_{m}} x$.

Using the finite transformation of the coordinate bases given by

$$
e_{m}^{\prime}\left(x^{\prime}\right)=e_{n}(x) \frac{\partial x^{n}}{\partial x^{\prime m}} \quad e^{\prime m}\left(x^{\prime}\right)=e^{n}(x) \frac{\partial x^{\prime m}}{\partial x^{n}}
$$

the finite transformation of the components of $\hat{W}$ are then

$$
\begin{gathered}
w^{\prime m}\left(x^{\prime}\right)=w^{n}(x) \frac{\partial x^{\prime m}}{\partial x^{n}}, \\
\hat{w}_{m n}^{\prime}\left(x^{\prime}\right)=\hat{w}_{p q}(x) \frac{\partial x^{p}}{\partial x^{\prime m}} \frac{\partial x^{q}}{\partial x^{\prime n}} .
\end{gathered}
$$

The finite transformation of the gerbe connection can be taken to be

$$
C_{(3)}^{\prime}\left(x^{\prime}\right)=C_{(3)}(x)+d \tilde{v}_{(2)}(x),
$$

so that

$$
\hat{w}_{(2)}^{\prime}\left(x^{\prime}\right)=\tilde{w}_{(2)}^{\prime}\left(x^{\prime}\right)+\imath_{w^{\prime}} C_{(3)}^{\prime}\left(x^{\prime}\right) \text {. }
$$

This then gives the finite transformation of $\tilde{w}_{(2)}$ :

$$
\begin{aligned}
\tilde{w}_{(2)}^{\prime}\left(x^{\prime}\right) & =\hat{w}_{(2)}^{\prime}\left(x^{\prime}\right)-\imath_{w^{\prime}} C_{(3)}^{\prime}\left(x^{\prime}\right), \\
& =\hat{w}_{(2)}(x)-\imath_{w}\left(C_{(3)}(x)+d \tilde{v}_{(2)}(x)\right), \\
& =\hat{w}_{(2)}(x)-\imath_{w} C_{(3)}(x)-\imath_{w} d \tilde{v}_{(2)}(x), \\
& =\tilde{w}_{(2)}(x)-\imath_{w} d \tilde{v}_{(2)}(x) .
\end{aligned}
$$

To summarize, the transformation of $W$ is given by

$$
\begin{gathered}
w^{\prime}\left(x^{\prime}\right)=w(x), \\
\tilde{w}_{(2)}^{\prime}\left(x^{\prime}\right)=\tilde{w}_{(2)}(x)-\imath_{w} d \tilde{v}_{(2)}(x),
\end{gathered}
$$

which implies the components $\left(w^{m}, \tilde{w}_{m n}\right)$ transform as

$$
\begin{gathered}
w^{\prime m}\left(x^{\prime}\right)=w^{n}(x) \frac{\partial x^{\prime m}}{\partial x^{n}} \\
\tilde{w}_{m n}^{\prime}\left(x^{\prime}\right)=\left(\tilde{w}_{p q}(x)-3 w^{r}(x)\left(d \tilde{v}_{(2)}\right)_{r p q}(x)\right) \frac{\partial x^{p}}{\partial x^{\prime m}} \frac{\partial x^{q}}{\partial x^{\prime n}},
\end{gathered}
$$

where $\left(d \tilde{v}_{(2)}\right)_{r p q}=\partial_{[r} \tilde{v}_{p q]}$.

\section{B. $S O(5,5)$ extended field theory}

In $S O(5,5)$ extended field theory [20,32], a generalized vector $W^{M}$ transforms as a spinor of $S O(5,5)$, where the indices $M, N=1, \ldots 16$ label the positive chirality spinor representation. It decomposes under $G L(5, \mathbb{R}) \subset S O(5,5)$ into

$$
W^{M}=\left(\begin{array}{c}
w^{m} \\
\tilde{w}_{m n} \\
\tilde{w}_{m n p q r}
\end{array}\right) \text {, }
$$

where $m, n=1, \ldots, 5, \tilde{w}_{m n}=-\tilde{w}_{n m}$, and $\tilde{w}_{m n p q r}=\tilde{w}_{[m n p q r]}$.

The coordinates in a patch $\mathcal{U}$ consist of 6 spacetime coordinates $y^{\mu}, \mu=0, \ldots, 5$, together with 16 internal coordinates $X^{M}$ transforming as a $\mathbf{1 6}$ of $S O(5,5)$. This decomposes under $G L(5, \mathbb{R}) \subset S O(5,5)$ into

$$
X^{M}=\left(\begin{array}{c}
x^{m} \\
\tilde{x}_{m n} \\
\tilde{x}_{m n p q r}
\end{array}\right) \text {, }
$$

where, in a suitable duality frame, $x^{m}$ are the usual coordinates on $T^{5}$ and $\tilde{x}_{m n}$ are periodic coordinates conjugate to M2-brane wrapping numbers on $T^{5}$ and $\tilde{x}_{m n p q r}$ are periodic coordinates conjugate to M5-brane wrapping numbers on $T^{5}$. Fields and gauge parameters depend on both $y^{\mu}$ and $X^{M}$, but we will suppress dependence on $y^{\mu}$ in what follows. 
The strong constraint of $S O(5,5)$ EFT is given by

$$
\gamma_{a}^{M N} \gamma_{P Q}^{a} \partial_{M}(\ldots) \partial_{N}(\ldots)=0
$$

where $(\ldots)$ represents fields and gauge parameters, $\gamma_{M N}^{a}$ is a gamma matrix of $S O(5,5)$ and $a=1, \ldots 10$ labels the vector representation of $S O(5,5)$. The strong constraint can be solved such that the fields are independent of wrapping coordinates $\tilde{x}_{m n}$ and $\tilde{x}_{m n p q r}$ so that

$$
\tilde{\partial}^{m n}(\ldots)=0 \quad \text { and } \quad \tilde{\partial}^{m n p q r}(\ldots)=0
$$

where $\tilde{\partial}^{m n}=\frac{\partial}{\partial \tilde{x}_{m n}}$ and $\tilde{\partial}^{m n p q r}=\frac{\partial}{\partial \tilde{x}_{m n p q r}}$. The gauge transformation of $S O(5,5)$ extended field theory is given by the generalized Lie derivative, which is

$$
\hat{\mathcal{L}}_{V} W^{M}=V^{N} \partial_{N} W^{M}-W^{N} \partial_{N} V^{M}+\frac{1}{2} \gamma_{a}^{M N} \gamma_{P Q}^{a} \partial_{N} V^{P} W^{Q} .
$$

It is convenient to rewrite the components of the generalized vector as

$$
\begin{gathered}
w=w^{m} e_{m}, \\
\tilde{w}_{(2)}=\frac{1}{2 !} \tilde{w}_{m n} e^{m} \wedge e^{n}, \\
\tilde{w}_{(5)}=\frac{1}{5 !} \tilde{w}_{m n p q r} e^{m} \wedge e^{n} \wedge e^{p} \wedge e^{q} \wedge e^{r} .
\end{gathered}
$$

Then the generalized vector $W$ is

$$
W=w \oplus \tilde{w}_{(2)} \oplus \tilde{w}_{(5)} .
$$

Under a gauge transformation with gauge parameter $V$, the generalized vector $W$ transforms as

$$
\delta_{V} W=\hat{\mathcal{L}}_{V} W,
$$

which decomposes into

$$
\begin{gathered}
\delta_{V} w=\mathcal{L}_{v} w, \\
\delta_{V} \tilde{w}_{(2)}=\mathcal{L}_{v} \tilde{w}_{(2)}-\iota_{w} d \tilde{v}_{(2)}, \\
\delta_{V} \tilde{w}_{(5)}=\mathcal{L}_{v} \tilde{w}_{(5)}-\tilde{w}_{(2)} \wedge d \tilde{v}_{(2)},
\end{gathered}
$$

where $\mathcal{L}_{v}$ is an ordinary Lie derivative with a parameter $v$.

Next we define a gerbe connection $C_{(3)}$,

$$
C_{(3)}=\frac{1}{3 !} C_{m n p} e^{m} \wedge e^{n} \wedge e^{p},
$$

which transforms under a gauge transformation as

$$
\delta_{V} C_{(3)}=\mathcal{L}_{v} C_{(3)}+d \tilde{v}_{(2)} .
$$

This allows us to define $\hat{w}_{(2)}$ and $\hat{w}_{(5)}$ by

$$
\begin{gathered}
\hat{w}_{(2)}=\tilde{w}_{(2)}+l_{w} C_{(3)}, \\
\hat{w}_{(5)}=\tilde{w}_{(5)}+\tilde{w}_{(2)} \wedge C_{(3)}+\frac{1}{2} l_{w} C_{(3)} \wedge C_{(3)} .
\end{gathered}
$$

Under a gauge transformation, these objects transform as a 2-form and a 5-form, respectively,

$$
\begin{aligned}
& \delta_{V} \hat{w}_{(2)}=\mathcal{L}_{v} \hat{w}_{(2)}, \\
& \delta_{V} \hat{w}_{(5)}=\mathcal{L}_{v} \hat{w}_{(5)},
\end{aligned}
$$

and are invariant under the $\tilde{v}$ transformations. Therefore, $\hat{W}=w \oplus \hat{w}_{(2)} \oplus \hat{w}_{(5)}$ is a section of $\left(T \oplus \Lambda^{2} T^{*} \oplus\right.$ $\left.\Lambda^{5} T^{*}\right) U$. The finite transformations are then

$$
\begin{aligned}
w^{\prime}\left(x^{\prime}\right) & =w(x), \\
\hat{w}_{(2)}^{\prime}\left(x^{\prime}\right) & =\hat{w}_{(2)}(x), \\
\hat{w}_{(5)}^{\prime}\left(x^{\prime}\right) & =\hat{w}_{(5)}(x),
\end{aligned}
$$

where $x^{\prime}=e^{-v^{m} \partial_{m}} x$.

Using the finite transformation of the coordinate bases given by

$$
e_{m}^{\prime}\left(x^{\prime}\right)=e_{n}(x) \frac{\partial x^{n}}{\partial x^{\prime m}} \quad e^{\prime m}\left(x^{\prime}\right)=e^{n}(x) \frac{\partial x^{\prime m}}{\partial x^{n}},
$$

the finite transformation of the components of $\hat{W}$ are

$$
\begin{gathered}
w^{\prime m}\left(x^{\prime}\right)=w^{n}(x) \frac{\partial x^{\prime m}}{\partial x^{n}}, \\
\hat{w}_{m n}^{\prime}\left(x^{\prime}\right)=\hat{w}_{m n}(x) \frac{\partial x^{p}}{\partial x^{\prime m}} \frac{\partial x^{q}}{\partial x^{\prime n}},
\end{gathered}
$$

$\hat{w}_{m n p q r}^{\prime}\left(x^{\prime}\right)=\hat{w}_{\text {stuvw }}(x) \frac{\partial x^{s}}{\partial x^{\prime m}} \frac{\partial x^{t}}{\partial x^{\prime n}} \frac{\partial x^{u}}{\partial x^{\prime p}} \frac{\partial x^{v}}{\partial x^{\prime q}} \frac{\partial x^{w}}{\partial x^{\prime r}}$.

The finite transformation of the gerbe connection can be taken to be

$$
C_{(3)}^{\prime}\left(x^{\prime}\right)=C_{(3)}(x)+d \tilde{v}_{(2)}(x),
$$

so that

$$
\begin{array}{r}
\hat{w}_{(2)}^{\prime}\left(x^{\prime}\right)=\tilde{w}_{(2)}^{\prime}\left(x^{\prime}\right)+l_{w^{\prime}} C_{(3)}^{\prime}\left(x^{\prime}\right) . \\
\hat{w}_{(5)}^{\prime}\left(x^{\prime}\right)=\tilde{w}_{(5)}^{\prime}\left(x^{\prime}\right)+\tilde{w}_{(2)}^{\prime}\left(x^{\prime}\right) \wedge C_{(3)}^{\prime}\left(x^{\prime}\right) \\
+\frac{1}{2} l_{w^{\prime}} C_{(3)}^{\prime}\left(x^{\prime}\right) \wedge C_{(3)}^{\prime}\left(x^{\prime}\right) .
\end{array}
$$


This give the finite transformation of $\tilde{w}_{(2)}$ :

$$
\begin{aligned}
\tilde{w}_{(2)}^{\prime}\left(x^{\prime}\right) & =\hat{w}_{(2)}^{\prime}\left(x^{\prime}\right)-\imath_{w^{\prime}} C_{(3)}^{\prime}\left(x^{\prime}\right) . \\
& =\hat{w}_{(2)}(x)-\imath_{w}\left(C_{(3)}(x)+d \tilde{v}_{(2)}(x)\right), \\
& =\hat{w}_{(2)}(x)-\imath_{w} C_{(3)}(x)-\imath_{w} d \tilde{v}_{(2)}(x), \\
& =\tilde{w}_{(2)}(x)-t_{w} d \tilde{v}_{(2)}(x) .
\end{aligned}
$$

Furthermore, the finite transformations of $\tilde{w}_{(5)}$ are

$$
\begin{aligned}
\tilde{w}_{(5)}^{\prime}\left(x^{\prime}\right)= & \hat{w}_{(5)}^{\prime}\left(x^{\prime}\right)-\tilde{w}_{(2)}^{\prime}\left(x^{\prime}\right) \wedge C_{(3)}^{\prime}\left(x^{\prime}\right)-\frac{1}{2} l_{w^{\prime}} C_{(3)}^{\prime}\left(x^{\prime}\right) \wedge C_{(3)}^{\prime}\left(x^{\prime}\right), \\
= & \left(\tilde{w}_{(5)}(x)+\tilde{w}_{(2)}(x) \wedge C_{(3)}(x)+\frac{1}{2} l_{w} C_{(3)}(x) \wedge C_{(3)}(x)\right) \\
& -\left(\tilde{w}_{(2)}(x)-l_{w} d \tilde{v}_{(2)}(x)\right) \wedge\left(C_{(3)}(x)+d \tilde{v}_{(2)}(x)\right) \\
& -\frac{1}{2} l_{w}\left(C_{(3)}(x)+d \tilde{v}_{(2)}(x)\right) \wedge\left(C_{(3)}(x)+d \tilde{v}_{(2)}(x)\right), \\
= & \tilde{w}_{(5)}(x)-\tilde{w}_{(2)}(x) \wedge d \tilde{v}_{(2)}(x)+\frac{1}{2} l_{w} d \tilde{v}_{(2)}(x) \wedge d \tilde{v}_{(2)}(x) .
\end{aligned}
$$

In summary, the transformation of $W$ is given by

$$
\begin{gathered}
w^{\prime}\left(x^{\prime}\right)=w(x), \\
\tilde{w}_{(2)}^{\prime}\left(x^{\prime}\right)=\tilde{w}_{(2)}(x)-l_{w} d \tilde{v}_{(2)}(x), \\
\tilde{w}_{(5)}^{\prime}\left(x^{\prime}\right)=\tilde{w}_{(5)}(x)-\tilde{w}_{(2)}(x) \wedge d \tilde{v}_{(2)}(x) \\
+\frac{1}{2} l_{w} d \tilde{v}_{(2)}(x) \wedge d \tilde{v}_{(2)}(x),
\end{gathered}
$$

which implies the components $\left(w^{m}, \tilde{w}_{m n}, \tilde{w}_{m n p q r}\right)$ transform as

$$
\begin{gathered}
w^{\prime m}\left(x^{\prime}\right)=w^{n}(x) \frac{\partial x^{\prime m}}{\partial x^{n}} \\
\tilde{w}_{m n}^{\prime}\left(x^{\prime}\right)=\left(\tilde{w}_{p q}(x)-3 w^{r}(x)\left(d \tilde{v}_{(2)}\right)_{r p q}(x)\right) \frac{\partial x^{p}}{\partial x^{\prime m}} \frac{\partial x^{q}}{\partial x^{\prime n}} \\
\tilde{w}_{m n p q r}^{\prime}\left(x^{\prime}\right)=\left(\tilde{w}_{s t u v w}(x)-30 \tilde{w}_{[s t}(x)\left(d \tilde{v}_{(2)}\right)_{u v w]}(x)\right. \\
\left.+15 w^{l}(x)\left(d \tilde{v}_{(2)}\right)_{l[s t}(x)\left(d \tilde{v}_{(2)}\right)_{u v w]}(x)\right) \\
\times \frac{\partial x^{s}}{\partial x^{\prime m}} \frac{\partial x^{t}}{\partial x^{\prime n}} \frac{\partial x^{u}}{\partial x^{\prime p}} \frac{\partial x^{v}}{\partial x^{\prime q}} \frac{\partial x^{w}}{\partial x^{\prime r}},
\end{gathered}
$$

where $\left(d \tilde{v}_{(2)}\right)_{r p q}=\partial_{[r} \tilde{v}_{p q]}$.

\section{C. $E_{6}$ extended field theory}

In $E_{6}$ extended field theory [24,26,35], a generalized vector $W^{M}$ transforms in the fundamental (27) representation of $E_{6}$ with $M, N=1, \ldots, 27$. It decomposes under $G L(6, \mathbb{R}) \subset E_{6}$ into

$$
W^{M}=\left(\begin{array}{c}
w^{m} \\
\tilde{w}_{m n} \\
\tilde{w}_{m n p q r}
\end{array}\right),
$$

where $m, n=1, \ldots, 6, \tilde{w}_{m n}=-\tilde{w}_{n m}$, and $\tilde{w}_{m n p q r}=\tilde{w}_{[m n p q r]}$.

The coordinates in a patch $\mathcal{U}$ consist of 5 spacetime coordinates $y^{\mu}, \mu=0, \ldots, 4$, together with 27 internal coordinates $X^{M}$ transforming as a $\mathbf{2 7}$ of $E_{6}$. This decomposes under $G L(6, \mathbb{R}) \subset E_{6}$ into

$$
X^{M}=\left(\begin{array}{c}
x^{m} \\
\tilde{x}_{m n} \\
\tilde{x}_{m n p q r}
\end{array}\right),
$$

where, in a suitable duality frame, $x^{m}$ are the usual coordinates on $T^{6}$ and $\tilde{x}_{m n}$ are periodic coordinates conjugate to M2-brane wrapping numbers on $T^{6}$ and $\tilde{x}_{\text {mnpqr }}$ are periodic coordinates conjugate to M5-brane wrapping numbers on $T^{6}$. Fields and gauge parameters depend on both $y^{\mu}$ and $X^{M}$, but we will suppress dependence on $y^{\mu}$ in what follows.

The strong constraint of $E_{6}$ EFT is given by

$$
c^{M N R} c_{P Q R} \partial_{M}(\ldots) \partial_{N}(\ldots)=0,
$$

where $(\ldots)$ represents fields and gauge parameters, and $c^{M N P}$ and $c_{M N P}$ are the $E_{6}$ invariant tensors. The strong constraint can be solved such that the fields are independent of wrapping coordinates $\tilde{x}_{m n}$ and $\tilde{x}_{m n p q r}$ so that

$$
\tilde{\partial}^{m n}(\ldots)=0 \quad \text { and } \quad \tilde{\partial}^{m n p q r}(\ldots)=0,
$$

where $\tilde{\partial}^{m n}=\frac{\partial}{\partial \tilde{x}_{m n}}$ and $\tilde{\partial}^{m n p q r}=\frac{\partial}{\partial \tilde{x}_{m n p q r}}$. The gauge transformation of $E_{6}$ extended field theory is given by the generalized Lie derivative, which is defined as 
$\hat{\mathcal{L}}_{V} W^{M}=V^{N} \partial_{N} W^{M}-W^{N} \partial_{N} V^{M}+10 c^{M N R} c_{P Q R} \partial_{N} V^{Q} W^{R}$,

It is convenient to rewrite the components of the generalized vector as

$$
\begin{gathered}
w=w^{m} e_{m}, \\
\tilde{w}_{(2)}=\frac{1}{2 !} \tilde{w}_{m n} e^{m} \wedge e^{n}, \\
\tilde{w}_{(5)}=\frac{1}{5 !} \tilde{w}_{\text {mnpqr }} e^{m} \wedge e^{n} \wedge e^{p} \wedge e^{q} \wedge e^{r} .
\end{gathered}
$$

Then the generalized vector $W$ is

$$
W=w \oplus \tilde{w}_{(2)} \oplus \tilde{w}_{(5)} .
$$

Under a gauge transformation with a gauge parameter $V$, the generalized vector $\mathrm{W}$ transform as

$$
\delta_{V} W=\hat{\mathcal{L}}_{V} W
$$

which decomposes into

$$
\begin{gathered}
\delta_{V} w=\mathcal{L}_{v} w \\
\delta_{V} \tilde{w}_{(2)}=\mathcal{L}_{v} \tilde{w}_{(2)}-\imath_{w} d \tilde{v}_{(2)}, \\
\delta_{V} \tilde{w}_{(5)}=\mathcal{L}_{v} \tilde{w}_{(5)}-\tilde{w}_{(2)} \wedge d \tilde{v}_{(2)}-\imath_{w} d \tilde{v}_{(5)},
\end{gathered}
$$

where $\mathcal{L}_{v}$ is an ordinary Lie derivative with a parameter $v$.

Next we introduce gerbe connections $C_{(3)}$ and $C_{(6)}$,

$$
\begin{gathered}
C_{(3)}=\frac{1}{3 !} C_{m n p} e^{m} \wedge e^{n} \wedge e^{p}, \\
C_{(6)}=\frac{1}{6 !} C_{m n p q r s} e^{m} \wedge e^{n} \wedge e^{p} \wedge e^{q} \wedge e^{r} \wedge e^{s},
\end{gathered}
$$

which transform under gauge transformation as

$$
\begin{gathered}
\delta_{V} C_{(3)}=\mathcal{L}_{v} C_{(3)}+d \tilde{v}_{(2)}, \\
\delta_{V} C_{(6)}=\mathcal{L}_{v} C_{(6)}+d \tilde{v}_{(5)}-\frac{1}{2} C_{(3)} \wedge d \tilde{v}_{(2)} .
\end{gathered}
$$

This allows us to define $\hat{w}_{(2)}$ and $\hat{w}_{(5)}$ by

$$
\begin{gathered}
\hat{w}_{(2)}=\tilde{w}_{(2)}+l_{w} C_{(3)}, \\
\hat{w}_{(5)}=\tilde{w}_{(5)}+\tilde{w}_{(2)} \wedge C_{(3)}+\frac{1}{2} l_{w} C_{(3)} \wedge C_{(3)}+l_{w} C_{(6)} .
\end{gathered}
$$

Under a gauge transformation, these objects transform as a 2 -form and a 5-form, respectively,

$$
\begin{aligned}
\delta_{V} \hat{w}_{(2)} & =\mathcal{L}_{v} \hat{w}_{(2)}, \\
\delta_{V} \hat{w}_{(5)} & =\mathcal{L}_{v} \hat{w}_{(5)},
\end{aligned}
$$

and are invariant under the $\tilde{v}$ transformation. Therefore, $\hat{W}=w \oplus \hat{w}_{(2)} \oplus \hat{w}_{(5)} \quad$ is a section of $\left(T \oplus \Lambda^{2} T^{*} \oplus\right.$ $\left.\Lambda^{5} T^{*}\right) U$. Their finite transformations are given by

$$
\begin{aligned}
w^{\prime}\left(x^{\prime}\right) & =w(x), \\
\hat{w}_{(2)}^{\prime}\left(x^{\prime}\right) & =\hat{w}_{(2)}(x), \\
\hat{w}_{(5)}^{\prime}\left(x^{\prime}\right) & =\hat{w}_{(5)}(x),
\end{aligned}
$$

where $x^{\prime}=e^{-v^{m} \partial_{m}} x$.

Using the finite transformation of the coordinate bases given by

$$
e_{m}^{\prime}\left(x^{\prime}\right)=e_{n}(x) \frac{\partial x^{n}}{\partial x^{\prime m}} \quad e^{\prime m}\left(x^{\prime}\right)=e^{n}(x) \frac{\partial x^{\prime m}}{\partial x^{n}},
$$

the finite transformations of the components of the $\hat{W}$ can be written as

$$
\begin{gathered}
w^{\prime m}\left(x^{\prime}\right)=w^{n}(x) \frac{\partial x^{\prime m}}{\partial x^{n}}, \\
\hat{w}_{m n}^{\prime}\left(x^{\prime}\right)=\hat{w}_{p q}(x) \frac{\partial x^{p}}{\partial x^{\prime m}} \frac{\partial x^{q}}{\partial x^{\prime n}}, \\
\hat{w}_{m n p q r}^{\prime}\left(x^{\prime}\right)=\hat{w}_{\text {stuvw }}(x) \frac{\partial x^{s}}{\partial x^{\prime m}} \frac{\partial x^{t}}{\partial x^{\prime n}} \frac{\partial x^{u}}{\partial x^{\prime p}} \frac{\partial x^{v}}{\partial x^{\prime q}} \frac{\partial x^{w}}{\partial x^{\prime r}} .
\end{gathered}
$$

The finite transformation of the gerbe connections can be taken to be

$$
C_{(3)}^{\prime}\left(x^{\prime}\right)=C_{(3)}(x)+d \tilde{v}_{(2)}(x),
$$

$C_{(6)}^{\prime}\left(x^{\prime}\right)=C_{(6)}(x)+d \tilde{v}_{(5)}(x)-\frac{1}{2} C_{(3)}(x) \wedge d \tilde{v}_{(2)}(x)$,

so that

$$
\begin{gathered}
\hat{w}_{(2)}^{\prime}\left(x^{\prime}\right)=\tilde{w}_{(2)}^{\prime}\left(x^{\prime}\right)+l_{w^{\prime}} C_{(3)}^{\prime}\left(x^{\prime}\right) \\
\hat{w}_{(5)}^{\prime}\left(x^{\prime}\right)=\tilde{w}_{(5)}^{\prime}\left(x^{\prime}\right)+\tilde{w}_{(2)}^{\prime}\left(x^{\prime}\right) \wedge C_{(3)}^{\prime}\left(x^{\prime}\right) \\
+\frac{1}{2} l_{w^{\prime}} C_{(3)}^{\prime}\left(x^{\prime}\right) \wedge C_{(3)}^{\prime}\left(x^{\prime}\right)+l_{w^{\prime}} C_{(6)}^{\prime}\left(x^{\prime}\right) .
\end{gathered}
$$

This then gives the finite transformation of $\tilde{w}_{(2)}$ :

$$
\begin{aligned}
& \tilde{w}_{(2)}^{\prime}\left(x^{\prime}\right)=\hat{w}_{(2)}^{\prime}\left(x^{\prime}\right)-\imath_{w^{\prime}} C_{(3)}^{\prime}\left(x^{\prime}\right) . \\
& =\hat{w}_{(2)}(x)-\imath_{w}\left(C_{(3)}(x)+d \tilde{v}_{(2)}(x)\right), \\
& =\hat{w}_{(2)}(x)-\imath_{w} C_{(3)}(x)-\imath_{w} d \tilde{v}_{(2)}(x), \\
& =\tilde{w}_{(2)}(x)-\imath_{w} d \tilde{v}_{(2)}(x),
\end{aligned}
$$

and the finite transformation of $\tilde{w}_{(5)}$ : 


$$
\begin{aligned}
\tilde{w}_{(5)}^{\prime}\left(x^{\prime}\right)= & \hat{w}_{(5)}^{\prime}\left(x^{\prime}\right)-\tilde{w}_{(2)}^{\prime}\left(x^{\prime}\right) \wedge C_{(3)}^{\prime}\left(x^{\prime}\right)-\frac{1}{2} l_{w^{\prime}} C_{(3)}^{\prime}\left(x^{\prime}\right) \wedge C_{(3)}^{\prime}\left(x^{\prime}\right)-l_{w^{\prime}} C_{(6)}^{\prime}\left(x^{\prime}\right), \\
= & \left(\tilde{w}_{(5)}(x)+\tilde{w}_{(2)}(x) \wedge C_{(3)}(x)+\frac{1}{2} l_{w} C_{(3)}(x) \wedge C_{(3)}(x)+l_{w} \tilde{C}_{(3)}(x)\right) \\
& -\left(\tilde{w}_{(2)}(x)-l_{w} d \tilde{v}_{(2)}(x)\right) \wedge\left(C_{(3)}(x)+d \tilde{v}_{(2)}(x)\right) \\
& -\frac{1}{2} l_{w}\left(C_{(3)}(x)+d \tilde{v}_{(2)}(x)\right) \wedge\left(C_{(3)}(x)+d \tilde{v}_{(2)}(x)\right) \\
& -i_{w}\left(C_{(6)}(x)+d \tilde{v}_{(5)}(x)-\frac{1}{2} C_{(3)}(x) \wedge d \tilde{v}_{(2)}(x)\right) \\
= & \tilde{w}_{(5)}(x)-\tilde{w}_{(2)}(x) \wedge d \tilde{v}_{(2)}(x)+\frac{1}{2} l_{w} d \tilde{v}_{(2)}(x) \wedge d \tilde{v}_{(2)}(x)-l_{w} d \tilde{v}_{(5)}(x) .
\end{aligned}
$$

In summary, the transformation of $W$ is given by

$$
\begin{gathered}
w^{\prime}\left(x^{\prime}\right)=w(x), \\
\tilde{w}_{(2)}^{\prime}\left(x^{\prime}\right)=\tilde{w}_{(2)}(x)-l_{w} d \tilde{v}_{(2)}(x), \\
\tilde{w}_{(5)}^{\prime}\left(x^{\prime}\right)=\tilde{w}_{(5)}(x)-\tilde{w}_{(2)}(x) \wedge d \tilde{v}_{(2)}(x) \\
+\frac{1}{2} l_{w} d \tilde{v}_{(2)}(x) \wedge d \tilde{v}_{(2)}(x)-l_{w} d \tilde{v}_{(5)}(x),
\end{gathered}
$$

which implies the components $\left(w^{m}, \tilde{w}_{m n}, \tilde{w}_{m n p q r}\right)$ transform as

$$
\begin{gathered}
w^{\prime m}\left(x^{\prime}\right)=w^{n}(x) \frac{\partial x^{\prime m}}{\partial x^{n}}, \\
\tilde{w}_{m n}^{\prime}\left(x^{\prime}\right)=\left(\tilde{w}_{p q}(x)-3 w^{r}(x)\left(d \tilde{v}_{(2)}\right)_{r p q}(x)\right) \frac{\partial x^{p}}{\partial x^{\prime m}} \frac{\partial x^{q}}{\partial x^{\prime n}} \\
\tilde{w}_{m n p q r}^{\prime}\left(x^{\prime}\right)= \\
+\tilde{w}_{s t u v w}(x)-30 \tilde{w}_{[s t}(x)\left(d \tilde{v}_{(2)}\right)_{u v w]}(x) \\
+15 w^{l}(x)\left(d \tilde{v}_{(2)}\right)_{l[s t}(x)\left(d \tilde{v}_{(2)}\right)_{u v w]}(x) \\
\left.-6 w^{l}(x)\left(d \tilde{v}_{(5)}\right)_{l s t u v w}(x)\right) \\
\\
\times \frac{\partial x^{s}}{\partial x^{\prime m}} \frac{\partial x^{t}}{\partial x^{\prime n}} \frac{\partial x^{u}}{\partial x^{\prime p}} \frac{\partial x^{v}}{\partial x^{\prime q}} \frac{\partial x^{w}}{\partial x^{\prime r}},
\end{gathered}
$$

where $\left(d \tilde{v}_{(2)}\right)_{r p q}=\partial_{[r} \tilde{v}_{p q]}$ and $\left(d \tilde{v}_{(5)}\right)_{m n p q r s}=\partial_{[m} \tilde{v}_{n p q r s]}$.

\section{GENERALIZED TENSORS}

In this section, we review the construction of tensors and twisted tensors in DFT of [12] and then generalize this to EFT.

\section{A. Generalized tensors of double field theory}

A generalized vector $W^{M}$ transforms as a vector under $O(D, D)$, so that under $G L(D, \mathbb{R}) \subset O(D, D)$ it transforms reducibly as

$$
W \rightarrow \hat{R}(\lambda) W
$$

where $\lambda^{m}{ }_{n} \in G L(D, \mathbb{R})$ and

$$
\hat{R}(\lambda)=\left(\begin{array}{cc}
\lambda & 0 \\
0 & \left(\lambda^{-1}\right)^{t}
\end{array}\right) .
$$

The untwisted version of $W$ is $\hat{W}^{M}$, which can be written as

$$
\hat{W}=L W
$$

where

$$
L=\left(\begin{array}{cc}
1 & 0 \\
-B & 1
\end{array}\right)
$$

denotes the matrix with components

$$
L_{N}^{M}=\left(\begin{array}{cc}
\delta^{m}{ }_{n} & 0 \\
-B_{m n} & \delta_{m}{ }^{n}
\end{array}\right)
$$

The transformation of the untwisted vector $\hat{W}$ is then

$$
\hat{W}^{\prime}\left(X^{\prime}\right)=\hat{R}(\Lambda) \hat{W}(X)
$$

where

$$
\Lambda_{n}^{m}=\frac{\partial x^{\prime m}}{\partial x^{n}} .
$$

The coordinate transformation acts only on the $x$ :

$$
X^{M} \rightarrow X^{\prime M}=\left(\begin{array}{c}
x^{\prime m} \\
\tilde{x}_{m}^{\prime}
\end{array}\right),
$$

with

$$
x^{m} \rightarrow x^{\prime m}(x), \quad \tilde{x}_{m} \rightarrow \tilde{x}_{m}^{\prime}=\tilde{x}_{m} .
$$

The transformation of the twisted vector $W$ was found by twisting the untwisted transformation and is 


$$
W^{\prime}\left(X^{\prime}\right)=R W(X)
$$

where

$$
R=L^{\prime}\left(X^{\prime}\right)^{-1} \hat{R}(\Lambda) L(X)=\hat{R}(\Lambda) S
$$

and

$$
L^{\prime}\left(X^{\prime}\right)=\left(\begin{array}{cc}
1 & 0 \\
-B^{\prime}\left(x^{\prime}\right) & 1
\end{array}\right)
$$

with $B^{\prime}\left(x^{\prime}\right)$ given by (2.24), and

$$
S=\left(\begin{array}{cc}
\delta^{m}{ }_{n} & 0 \\
2 \partial_{[m} \tilde{v}_{n]} & \delta_{m}{ }^{n}
\end{array}\right) .
$$

The matrices $R, \hat{R}, L, S$ are all in $O(D, D)$.

Lowering indices with $\eta$ gives similar formulas for a generalized vector with lower index

$$
U_{M}=\left(\begin{array}{c}
\tilde{u}_{m} \\
u^{m}
\end{array}\right)
$$

The untwisted vector

$$
\hat{U}_{M}=\left(\begin{array}{c}
\hat{u}_{m} \\
u^{m}
\end{array}\right)=\left(\begin{array}{c}
\tilde{u}_{m}-B_{m n} u^{n} \\
u^{m}
\end{array}\right)
$$

transforms with

$$
\delta \hat{U}_{M}=\mathcal{L}_{v} \hat{U}_{M}
$$

and is invariant under $\tilde{v}$ transformations. Then the untwisted vector is

$$
\hat{U}=U L^{-1}
$$

[i.e. $\hat{U}_{M}=U_{N}\left(L^{-1}\right)^{N}{ }_{M}$; recall $\eta L \eta^{-1}=\left(L^{t}\right)^{-1}$ as $L$ is in $O(D, D)]$ and transforms under a finite transformation as

$$
\hat{U}^{\prime}\left(X^{\prime}\right)=\hat{U}(X) \hat{R}^{-1}
$$

where here and in what follows $\hat{R}=\hat{R}(\Lambda)$. For the twisted vectors

$$
U^{\prime}\left(X^{\prime}\right)=U(X) R^{-1} .
$$

This extends to arbitrary generalized tensors $T^{M N \ldots}{ }_{P Q \ldots}$. We define the untwisted tensor

$$
\hat{T}^{M N \ldots}{ }_{P Q \ldots}=L^{M}{ }_{R} L^{N}{ }_{S} \ldots T^{R S \ldots}{ }_{T U \ldots}\left(L^{-1}\right)^{T}{ }_{P}\left(L^{-1}\right)^{U}{ }_{Q} \ldots
$$

which transforms as
$\hat{T}^{\prime M N \ldots}{ }_{P Q \ldots}\left(X^{\prime}\right)=\hat{R}^{M}{ }_{R} \hat{R}^{N}{ }_{S} \ldots T^{R S \ldots}{ }_{T U \ldots}\left(\hat{R}^{-1}\right)^{T}{ }_{P}\left(\hat{R}^{-1}\right)^{U}{ }_{Q} \ldots$

so that the original tensor transforms as

$T^{\prime M N \ldots}{ }_{P Q \ldots}\left(X^{\prime}\right)=R^{M}{ }_{R} R^{N}{ }_{S} \ldots T^{R S \ldots} T U \ldots\left(R^{-1}\right)^{T}{ }_{P}\left(R^{-1}\right)^{U}{ }_{Q} \ldots$

Raising all lower indices with $\eta$ gives a generalized tensor $T^{M_{1} \ldots M_{p}}$ of some rank $p$ which is a section of $E^{p}$ while $\hat{T}^{M_{1} \ldots M_{p}}$ is a section of $\left(T \oplus T^{*}\right)^{p}$. In particular,

$$
\hat{\eta}_{M N}=\eta_{M N}
$$

as $L \in O(D, D)$, and is invariant, $\eta^{\prime}=\eta$.

\section{B. Generalized tensors of extended field theory}

For each of the extended field theories, a similar structure applies, with matrices $L, L^{\prime}, R, \hat{R}, S$, all of which are in the duality group $G$ which is $S L(5, \mathbb{R}), S O(5,5)$ or $E_{6}$. The untwisted form $\hat{W}^{M}$ of a generalized vector $W^{M}$ can be written as

$$
\hat{W}=L W
$$

The generalized vector transforms as a representation of $G$ and decompose into a reducible representation of $G L(d, \mathbb{R})$ (where $d$ is the rank of $G$ ) under which $\lambda \in G L(d, \mathbb{R})$ acts on $W$ to give $W \rightarrow \hat{R}(\lambda) W$. The transformation of the untwisted vector $\hat{W}$ is then

$$
\hat{W}^{\prime}\left(X^{\prime}\right)=\hat{R}(\lambda) \hat{W}(X)
$$

where

$$
\Lambda_{n}^{m}=\frac{\partial x^{\prime m}}{\partial x^{n}} .
$$

The transformation of the twisted vector $W$ was found by twisting the untwisted transformation and can be written as

$$
W^{\prime}\left(X^{\prime}\right)=R W(X)
$$

where

$$
R=L^{\prime}\left(X^{\prime}\right)^{-1} \hat{R}(\Lambda) L(X)=\hat{R}(\Lambda) S
$$

where $L^{\prime}$ generates shifts of the antisymmetric tensor gauge fields and $S$ is the corresponding gauge transformation; see below for explicit forms.

As for the DFT case, this extends to arbitrary generalized tensors $T^{M N \ldots} P Q \ldots$. We define the untwisted tensor 
$\hat{T}^{M N \ldots}{ }_{P Q} \ldots=L^{M}{ }_{R} L^{N}{ }_{S} \ldots T^{R S \ldots}{ }_{T U \ldots}\left(L^{-1}\right)^{T}{ }_{P}\left(L^{-1}\right)^{U}{ }_{Q} \ldots$

which transforms as

$$
\hat{T}^{\prime M N \ldots}{ }_{P Q \ldots}\left(X^{\prime}\right)=\hat{R}^{M}{ }_{R} \hat{R}^{N}{ }_{S} \ldots T^{R S \ldots}{ }_{T U \ldots}\left(\hat{R}^{-1}\right)^{T}{ }_{P}\left(\hat{R}^{-1}\right)^{U}{ }_{Q} \ldots
$$

so that the original tensor transforms as
$T^{\prime M N \ldots}{ }_{P Q \ldots}\left(X^{\prime}\right)=R^{M}{ }_{R} R^{N}{ }_{S} \ldots T^{R S \ldots}{ }_{T U \ldots}\left(R^{-1}\right)^{T}{ }_{P}\left(R^{-1}\right)^{U}{ }_{Q} \ldots$

We now give the explicit forms of the matrices appearing above. For the $S L(5, \mathbb{R})$ case, the untwisted vector is

$$
\hat{W}^{M}=L^{M}{ }_{N} W^{N}=\left(\begin{array}{cc}
\delta^{m}{ }_{l} & 0 \\
C_{l m n} & \delta_{m n} p q
\end{array}\right)\left(\begin{array}{c}
w^{l} \\
\tilde{w}_{p q}
\end{array}\right),
$$

where $\delta_{m n}{ }^{p q}=\frac{1}{2}\left(\delta_{m}{ }^{p} \delta_{n}{ }^{q}-\delta_{m}{ }^{q} \delta_{n}{ }^{p}\right)$. For the $S O(5,5)$ case, the untwisted vector is

$$
\hat{W}^{M}=L^{M}{ }_{N} W^{N}=\left(\begin{array}{ccc}
\delta^{m}{ }_{l} & 0 & 0 \\
C_{l m n} & \delta_{m n}{ }^{p q} & 0 \\
5 C_{l[m n} C_{p q r]} & 10 \delta_{[m n}{ }^{p q} C_{r s t]} & \delta_{\text {mnpqr }}{ }_{\text {stuvw }}
\end{array}\right)\left(\begin{array}{c}
w^{l} \\
\tilde{w}_{p q} \\
\tilde{w}_{s t u v w}
\end{array}\right) \text {, }
$$

where $\delta_{m n p q r}{ }^{\text {stuvw }}=\delta_{[m}{ }^{s} \ldots \delta_{r]}{ }^{w}$. For $E_{6}$ case, the untwisted vector is

$$
\hat{W}^{M}=L^{M}{ }_{N} W^{N}=\left(\begin{array}{ccc}
\delta^{m}{ }_{l} & 0 & 0 \\
C_{l m n} & \delta_{m n}^{p q} & 0 \\
C_{l m n p q r}+5 C_{l[m n} C_{p q r]} & 10 \delta_{[m n}^{p q} C_{r s t]} & \delta_{\text {mnpqr }}{ }^{\text {stuvw }}
\end{array}\right)\left(\begin{array}{c}
w^{l} \\
\tilde{w}_{p q} \\
\tilde{w}_{\text {stuvw }}
\end{array}\right) .
$$

Then the $L$ matrix for the $S L(5, \mathbb{R})$ theory is

$$
L^{M}{ }_{N}=\left(\begin{array}{cc}
\delta^{m}{ }_{l} & 0 \\
C_{l m n} & \delta_{m n}{ }^{p q}
\end{array}\right)
$$

while for the $S O(5,5)$ theory it is

$$
L^{M}{ }_{N}=\left(\begin{array}{ccc}
\delta^{m}{ }_{l} & 0 & 0 \\
C_{l m n} & \delta_{m n}{ }^{p q} & 0 \\
5 C_{l[m n} C_{p q r]} & 10 \delta_{[m n}{ }^{p q} C_{r s t]} & \delta_{m n p q r}{ }^{\text {stuvw }}
\end{array}\right)
$$

and for the $E_{6}$ theory it is

$$
L^{M}{ }_{N}=\left(\begin{array}{ccc}
\delta^{m}{ }_{l} & 0 & 0 \\
C_{l m n} & \delta_{m n}{ }^{p q} & 0 \\
C_{l m n p q r}+5 C_{l[m n} C_{p q r]} & 10 \delta_{[m n}^{p q} C_{r s t]} & \delta_{m n p q r}^{\text {stuvw }}
\end{array}\right) .
$$

The matrices $\hat{R}$ for the $S L(5, \mathbb{R}), S O(5,5)$ and $E_{6}$ theories are given by

$$
\begin{gathered}
\hat{R}^{M}{ }_{N}=\left(\begin{array}{cc}
\Lambda^{m}{ }_{l} & 0 \\
0 & \left(\Lambda^{-1}\right)_{m}{ }^{p}\left(\Lambda^{-1}\right)_{n}{ }^{q}
\end{array}\right) \\
\hat{R}^{M}{ }_{N}=\left(\begin{array}{ccc}
\Lambda^{m}{ }_{l} & 0 & 0 \\
0 & \left(\Lambda^{-1}\right)_{m}{ }^{p}\left(\Lambda^{-1}\right)_{n}{ }^{q} & 0 \\
0 & 0 & \left(\Lambda^{-1}\right)_{m}{ }^{s}\left(\Lambda^{-1}\right)_{n}{ }^{t}\left(\Lambda^{-1}\right)_{p}{ }^{u}\left(\Lambda^{-1}\right)_{q}{ }^{v}\left(\Lambda^{-1}\right)_{r}{ }^{w}
\end{array}\right),
\end{gathered}
$$




$$
\hat{R}^{M}{ }_{N}=\left(\begin{array}{ccc}
\Lambda^{m}{ }_{l} & 0 & 0 \\
0 & \left(\Lambda^{-1}\right)_{m}{ }^{p}\left(\Lambda^{-1}\right)_{n}{ }^{q} & 0 \\
0 & 0 & \left(\Lambda^{-1}\right)_{m}{ }^{s}\left(\Lambda^{-1}\right)_{n}{ }^{t}\left(\Lambda^{-1}\right)_{p}{ }^{u}\left(\Lambda^{-1}\right)_{q}{ }^{v}\left(\Lambda^{-1}\right)_{r}{ }^{w}
\end{array}\right)
$$

respectively.

The $L^{\prime}$ matrix for the $S L(5, \mathbb{R})$ theory is

$$
L^{\prime M}{ }_{N}\left(X^{\prime}\right)=\left(\begin{array}{cc}
\delta^{m}{ }_{l} & 0 \\
C_{l m n}^{\prime}\left(X^{\prime}\right) & \delta_{m n}{ }^{p q}
\end{array}\right),
$$

while for the $S O(5,5)$ theory it is

$$
L^{\prime M}{ }_{N}\left(X^{\prime}\right)=\left(\begin{array}{ccc}
\delta^{m}{ }_{l} & 0 & 0 \\
C_{l m n}^{\prime}\left(X^{\prime}\right) & \delta_{m n}{ }^{p q} & 0 \\
5 C_{l[m n}^{\prime}\left(X^{\prime}\right) C_{p q r]}^{\prime}\left(X^{\prime}\right) & 10 \delta_{[m n}{ }^{p q} C_{r s t]}^{\prime}\left(X^{\prime}\right) & \delta_{\text {mnpqr }}{ }_{\text {stuvw }}
\end{array}\right)
$$

and for the $E_{6}$ theory it is

$$
L^{\prime M}{ }_{N}\left(X^{\prime}\right)=\left(\begin{array}{ccc}
\delta^{m}{ }_{l} & 0 & 0 \\
C_{l m n}^{\prime}\left(X^{\prime}\right) & \delta_{m n}^{p q} & 0 \\
C_{l m n p q r}^{\prime}\left(X^{\prime}\right)+5 C_{l[m n}^{\prime}\left(X^{\prime}\right) C_{p q r]}^{\prime}\left(X^{\prime}\right) & 10 \delta_{[m n}{ }^{p q} C_{r s t]}^{\prime}\left(X^{\prime}\right) & \delta_{m n p q r}{ }^{\text {stuvw }}
\end{array}\right) .
$$

Finally, the matrix $S$ for the $S L(5, \mathbb{R})$ theory is

$$
S^{M}{ }_{N}=\left(\begin{array}{cc}
\delta^{m}{ }_{l} & 0 \\
-3\left(d \tilde{v}_{(2)}\right)_{l m n} & \delta_{m n}{ }^{p q}
\end{array}\right)
$$

while for the $S O(5,5)$ theory it is

$$
S^{M}{ }_{N}=\left(\begin{array}{ccc}
\delta^{m}{ }_{l} & 0 & 0 \\
-3\left(d \tilde{v}_{(2)}\right)_{l m n} & \delta_{m n}{ }^{p q} & 0 \\
15\left(d \tilde{v}_{(2)}\right)_{l[m n}\left(d \tilde{v}_{(2)}\right)_{p q r]} & -30 \delta_{[m n}^{p q} \partial_{r} \tilde{v}_{s t]} & \delta_{m n p q r}^{\text {stuvw }}
\end{array}\right)
$$

and for the $E_{6}$ theory it is

$$
S^{M}{ }_{N}=\left(\begin{array}{ccc}
\delta^{m}{ }_{l} & 0 & 0 \\
-3\left(d \tilde{v}_{(2)}\right)_{l m n} & \delta_{m n}{ }^{p q} & 0 \\
-6\left(d \tilde{v}_{(5)}\right)_{l m n p q r}+15\left(d \tilde{v}_{(2)}\right)_{l[m n}\left(d \tilde{v}_{(2)}\right)_{p q r]} & -30 \delta_{[m n}{ }^{p q} \partial_{r} \tilde{v}_{s t]} & \delta_{m n p q r}{ }_{\text {stuvw }}
\end{array}\right)
$$

where $\left(d \tilde{v}_{(2)}\right)_{r p q}=\partial_{[r} \tilde{v}_{p q]}$ and $\left(d \tilde{v}_{(5)}\right)_{m n p q r s}=\partial_{[m} \tilde{v}_{n p q r s]}$.

\section{THE GENERALIZED METRIC IN DFT AND EFT}

For DFT and EFT, there is a duality group $G$ [which is $O(D, D)$ for DFT and $E_{D}$ for EFT] with maximal compact subgroup $H$. Remarkably, the fields $\left(g_{m n}, B_{m n}\right)$ in DFT and $\left(g_{m n}, C_{m n p}, C_{m n p q r s}\right)$ in EFT can be regarded as a field taking values in the coset $G / H$-the coset space can be locally parametrised by $g_{m n}, B_{m n}$ or $g_{m n}, C_{m n p}, C_{m n p q r s}$ [16]. A field taking values in the coset $G / H$ can be represented by a vielbein $\mathcal{V}^{A}{ }_{M}(X) \in G$ transforming as

$$
\mathcal{V} \rightarrow h \mathcal{V} g
$$

under a rigid $G$ transformation $g^{N}{ }_{M} \in G$ and a local $H$ transformation $h^{A}{ }_{B}(X) \in H$. If $k_{A B}$ is an $H$-invariant metric 
( $h^{t} k h=k$ for all $h \in H$ ) then the degrees of freedom can also be encoded in a generalized metric

$$
\mathcal{H}_{M N}=k_{A B} \mathcal{V}^{A}{ }_{M} \mathcal{V}^{B}{ }_{N}
$$

which by construction is invariant under $H$ transformations.

We now show how the coset is parametrized by the fields $\left(g_{m n}, B_{m n}\right)$ or $\left(g_{m n}, C_{m n p}, C_{m n p q r s}\right)$. Let $e_{m}^{a}$ be a vielbein for the metric $g_{m n}$, with

$$
g_{m n}=\delta_{a b} e^{a}{ }_{m} e^{b}
$$

and inverse vielbein $e_{a}{ }^{m}$. The indices $a, b$ transform under the tangent space group $O(D)$. Then the vielbein $\mathcal{V}$ can be written in terms of $e^{a}{ }_{m}$ and $\left(g_{m n}, B_{m n}\right)$ or $\left(g_{m n}, C_{m n p}, C_{m n p q r s}\right)$ as

$$
\mathcal{V}=h \hat{R}\left(e^{a}{ }_{m}\right) L
$$

(This can be viewed as a consequence of the Iwasawa decomposition theorem.) Here $h \in H$ and can be chosen to be $h=1$ by a local $H$ transformation. The dependence on the gauge fields is given by the matrix $L(B)$ in DFT and $L\left(C_{(3)}, C_{(6)}\right)$ in EFT; $L$ is of the form $L=1+N$ where $N$ is lower triangular. Finally $\hat{R}(e)$ is the matrix $\hat{R}(\lambda)$ given above, with $\lambda^{a}{ }_{m}=e^{a}{ }_{m}$ and serves to convert "curved" indices $m, n$ to "flat" indices $a, b$.

Then the generalized metric is given by

$$
\mathcal{H}(V, W)=\hat{\mathcal{H}}(\hat{V}, \hat{W})
$$

for any generalized vectors $V, W$, where

$$
\hat{\mathcal{H}}(\hat{V}, \hat{W})=k(\hat{R}(e) \hat{V}, \hat{R}(e) \hat{W}) .
$$

Explicitly,

$$
\hat{\mathcal{H}}_{M N}=k_{A B} \hat{R}(e)^{A}{ }_{M} \hat{R}(e)^{B}{ }_{N}
$$

and

$$
\mathcal{H}_{M N}=\hat{\mathcal{H}}_{P Q} L^{P}{ }_{M} L^{Q}{ }_{N} .
$$

Then $\hat{\mathcal{H}}_{P Q}$ is the untwisted form of the generalized metric, and is the natural metric on generalized or extended tangent vectors induced by the metric $g$ for ordinary vectors.

We now show how this works for the cases discussed here. For DFT, we recover the discussion of [12]. An untwisted vector decomposes as

$$
\hat{W}^{M}=\left(\begin{array}{c}
w^{m} \\
\hat{w}_{m}
\end{array}\right),
$$

so

$$
\hat{R}(e) \hat{W}=\left(\begin{array}{c}
w^{a} \\
\hat{w}_{a}
\end{array}\right)
$$

where as usual

$$
w^{a}=e^{a}{ }_{m} w^{m}, \quad \hat{w}_{a}=e_{a}{ }^{m} \hat{w}_{m} .
$$

The metric $k_{A B}$ decomposes under $O(D)$ to give

$$
k_{A B}=\left(\begin{array}{cc}
\delta_{a b} & 0 \\
0 & \delta^{a b}
\end{array}\right)
$$

so

$$
k(\hat{R}(e) \hat{V}, \hat{R}(e) \hat{W})=\delta_{a b} v^{a} w^{b}+\delta^{a b} \hat{v}_{a} \hat{w}_{b} .
$$

This is equal to $\hat{\mathcal{H}}(\hat{V}, \hat{W})=\hat{\mathcal{H}}_{M N} \hat{V}^{M} \hat{W}^{N}$ so

$$
\hat{\mathcal{H}}_{M N}=\left(\begin{array}{cc}
g_{m n} & 0 \\
0 & g^{m n}
\end{array}\right)
$$

which is the standard metric on $T \oplus T^{*}$ induced by the metric $g$ on $T$. Then the generalized metric is

$$
\mathcal{H}_{M N}=\hat{\mathcal{H}}_{P Q} L^{P}{ }_{M} L^{Q}{ }_{N}
$$

where $L(B)$ is given by (4.4). This gives the standard result

$$
\mathcal{H}_{M N}=\left(\begin{array}{cc}
g_{m n}-B_{m k} g^{k l} B_{l n} & B_{m k} g^{k n} \\
-g^{m k} B_{k n} & g^{m n}
\end{array}\right) .
$$

Consider now EFT with $G=E_{D}$ for $D=5$, 6. An untwisted vector decomposes as

$$
\hat{W}^{M}=\left(\begin{array}{c}
w^{m} \\
\hat{w}_{m n} \\
\hat{w}_{\text {mnpqr }}
\end{array}\right),
$$

so

$$
\hat{R}(e) \hat{W}=\left(\begin{array}{c}
w^{a} \\
\hat{w}_{a b} \\
\hat{w}_{a b c d e}
\end{array}\right),
$$

with all indices converted to tangent space indices using $e^{a}{ }_{m}$. The metric $k_{A B}$ decomposes under $O(D)$ to give

$$
k(\hat{R}(e) \hat{V}, \hat{R}(e) \hat{W})=v^{a} w_{a}+\frac{1}{2} \hat{v}_{a b} \hat{w}^{a b}+\frac{1}{5 !} \hat{v}_{a b c d e} \hat{w}^{a b c d e}
$$

where indices have been raised or lowered with $\delta_{a b}$. The matrix can then be written as 


$$
k_{A B}=\left(\begin{array}{ccc}
\delta_{a b} & 0 & \\
0 & \delta^{a b, c d} & 0 \\
0 & 0 & \delta^{a_{1} \ldots a_{5}, b_{1} \ldots b_{5}}
\end{array}\right)
$$

where $\delta^{a b, c d}=\frac{1}{2} \delta^{c[a} \delta^{b] d}$ and a similar expression for $\delta^{a_{1} \ldots a_{5}, b_{1} \ldots b_{5}}$. Then $(5.20)$ is equal to $\hat{\mathcal{H}}(\hat{V}, \hat{W})=$ $\hat{\mathcal{H}}_{M N} \hat{V}^{M} \hat{W}^{N}$ so

$$
\hat{\mathcal{H}}_{M N} \hat{V}^{M} \hat{W}^{N}=v^{m} w_{m}+\frac{1}{2} \hat{v}_{m n} \hat{w}^{m n}+\frac{1}{5 !} \hat{v}_{\text {mnpqr }} \hat{w}^{\text {mnpqr }}
$$

where indices have been raised and lowered with $g_{m n}$. The corresponding matrix is

$$
\hat{\mathcal{H}}_{M N}=\left(\begin{array}{ccc}
g_{m n} & 0 & \\
0 & g^{m n, p q} & 0 \\
0 & 0 & g^{m_{1} \ldots m_{5}, n_{1} \ldots n_{5}}
\end{array}\right)
$$

where $g^{l k, m n}=\frac{1}{2} g^{m[l} g^{k] n}$ and a similar expression for $g^{a_{1} \ldots a_{5}, b_{1} \ldots b_{5}}$. Then $\hat{\mathcal{H}}_{M N}$ is the standard metric on $T \oplus \Lambda^{2} T^{*} \oplus \Lambda^{5} T^{*}$ induced by the metric $g$ on $T$.

The generalized metric is then the twisted from of this

$$
\mathcal{H}_{M N}=\hat{\mathcal{H}}_{P Q} L^{P}{ }_{M} L^{Q}{ }_{N}
$$

where $L\left(C_{(3)}, C_{(6)}\right)$ is given by (4.36) or (4.37). This then gives explicit forms for the generalized metric, in agreement with [16,20,24,26,32,33,35].

For $S L(5, \mathbb{R})$, there is no $C_{(6)}$ or $\hat{w}_{(5)}$, but similar formulas apply with

$$
\hat{\mathcal{H}}_{M N}=\left(\begin{array}{cc}
g_{m n} & 0 \\
0 & g^{k l, p q}
\end{array}\right)
$$

and (5.8) with $L\left(C_{(3)}\right)$ given by (4.35) gives the

$$
\mathcal{H}_{M N}=\left(\begin{array}{cc}
g_{m n}+C_{m}{ }^{r s} C_{r s n} & \frac{1}{2} C_{m}{ }^{p q} \\
\frac{1}{2} C^{k l}{ }_{n} & g^{k l, p q}
\end{array}\right),
$$

recovering the generalized metric given in [16].

\section{ACKNOWLEDGMENTS}

The work of CMH was supported by STFC Grant No. ST/L00044X/1 and EPSRC Grant No. EP/K034456/1.
[1] C. Hull and B. Zwiebach, Double field theory, J. High Energy Phys. 09 (2009) 099.

[2] O. Hohm, C. Hull, and B. Zwiebach, Background independent action for double field theory, J. High Energy Phys. 07 (2010) 016.

[3] O. Hohm, C. Hull, and B. Zwiebach, Generalised metric formulation of double field theory, J. High Energy Phys. 08 (2010) 008.

[4] W. Siegel, Superspace duality in low-energy superstrings, Phys. Rev. D 48, 2826 (1993).

[5] N. Hitchin, Generalised Calabi-Yau manifolds, Quart. J. Math. Oxford Ser. 54, 281 (2003).

[6] M. Gualtieri, Generalized complex geometry, arXiv:math/ 0401221.

[7] N. Hitchin, Brackets, forms and invariant functionals, arXiv: math/0508618.

[8] N. Hitchin, Lectures on generalized geometry, arXiv:1008.0973.

[9] M. Grana, R. Minasian, M. Petrini, and D. Waldram, T-duality, generalized geometry and non-geometric backgrounds, J. High Energy Phys. 04 (2009) 075.

[10] A. Coimbra, C. Strickland-Constable, and D. Waldram, Supergravity as generalised geometry I: Type II theories, J. High Energy Phys. 11 (2011) 091.

[11] K. Lee, C. Strickland-Constable, and D. Waldram, New gaugings and non-geometry, arXiv:1506.03457.
[12] C. M. Hull, Finite gauge transformations and geometry in double field theory, J. High Energy Phys. 04 (2015) 109.

[13] G. Aldazabal, D. Marques, and C. Nunez, Double field theory: A pedagogical review, Classical Quantum Gravity 30, 163001 (2013).

[14] D. S. Berman and D. C. Thompson, Duality Symmetric String and M-Theory, arXiv:1306.2643.

[15] O. Hohm, D. Lust, and B. Zwiebach, The spacetime of double field theory: Review, remarks, and outlook, Fortsch. Phys. 61, 926 (2013).

[16] C. M. Hull, Generalised geometry for M-theory, J. High Energy Phys. 07 (2007) 079.

[17] P. P. Pacheco and D. Waldram, M-theory, exceptional generalised geometry and superpotentials, J. High Energy Phys. 09 (2008) 123.

[18] C. M. Hull, A geometry for non-geometric string backgrounds, J. High Energy Phys. 10 (2005) 065.

[19] D. S. Berman and M. J. Perry, Generalized geometry and M theory, J. High Energy Phys. 06 (2011) 074.

[20] D. S. Berman, H. Godazgar, and M. J. Perry, SO (5, 5) duality in M-theory and generalized geometry, Phys. Lett. B 700, 65 (2011).

[21] D. S. Berman, H. Godazgar, M. Godazgar, and M. J. Perry, The local symmetries of M-theory and their formulation in generalised geometry, J. High Energy Phys. 01 (2012) 012 
[22] M. Cederwall, J. Edlund, and A. Karlsson, Exceptional geometry and tensor fields, J. High Energy Phys. 07 (2013) 028.

[23] O. Hohm and H. Samtleben, Exceptional Form of D $=11$ Supergravity, Phys. Rev. Lett. 111 (2013) 231601.

[24] O. Hohm and H. Samtleben, Exceptional field theory I: $E_{6(6)}$ covariant form of M-theory and type IIB, Phys. Rev. D 89, 066016 (2014).

[25] O. Hohm and H. Samtleben, Exceptional field theory. II. $\mathrm{E}_{7(7)}$, Phys. Rev. D 89, 066017 (2014).

[26] E. T. Musaev, Exceptional field theory for $E_{6(6)}$ supergravity, TSPU Bulletin 12, 198 (2014).

[27] H. Godazgar, M. Godazgar, O. Hohm, H. Nicolai, and H. Samtleben, Supersymmetric $\mathrm{E}_{7(7)}$ exceptional field theory, J. High Energy Phys. 09 (2014) 044.

[28] O. Hohm and H. Samtleben, Exceptional field theory. III. $\mathrm{E}_{8(8)}$, Phys. Rev. D 90, 066002 (2014).

[29] J. A. Rosabal, On the exceptional generalised Lie derivative for $d \geq 7$, J. High Energy Phys. 09 (2015) 153.

[30] C. D. A. Blair and E. Malek, Geometry and fluxes of SL(5) exceptional field theory, J. High Energy Phys. 03 (2015) 144.

[31] D. S. Berman and F. J. Rudolph, Strings, branes and the selfdual solutions of exceptional field theory, J. High Energy Phys. 05 (2015) 130.

[32] A. Abzalov, I. Bakhmatov, and E. T. Musaev, Exceptional field theory: SO(5, 5), J. High Energy Phys. 06 (2015) 088.
[33] D. S. Berman, H. Godazgar, M. J. Perry, and P. West, Duality invariant actions and generalised geometry, J. High Energy Phys. 02 (2012) 108.

[34] M. Cederwall and J. A. Rosabal, $E_{8}$ geometry, J. High Energy Phys. 07 (2015) 007.

[35] A. Baguet, O. Hohm, and H. Samtleben, $\mathrm{E}_{6(6)}$ exceptional field theory: Review and embedding of type IIB, Proc. Sci., CORFU 2014 (2015) 133 [arXiv:1506.01065].

[36] A. G. Tumanov and P. West, E11 and exceptional field theory, arXiv:1507.08912.

[37] G. Bossard and A. Kleinschmidt, Loops in exceptional field theory, J. High Energy Phys. 01 (2016) 164.

[38] O. Hohm and B. Zwiebach, Large gauge transformations in double field theory, J. High Energy Phys. 02 (2013) 075.

[39] J.-H. Park, Comments on double field theory and diffeomorphisms, J. High Energy Phys. 06 (2013) 098.

[40] D. S. Berman, M. Cederwall, and M. J. Perry, Global aspects of double geometry, J. High Energy Phys. 09 (2014) 066.

[41] G. Papadopoulos, Seeking the balance: Patching double and exceptional field theories, J. High Energy Phys. 10 (2014) 089.

[42] M. Cederwall, The geometry behind double geometry, J. High Energy Phys. 09 (2014) 070.

[43] G. Papadopoulos, C-spaces, generalized geometry and double field theory, J. High Energy Phys. 09 (2015) 029.

[44] S. J. Rey and Y. Sakatani, Finite transformations in doubled and exceptional space, arXiv:1510.06735. 